\title{
An Effective Deep Learning Approach Based On CNN to Predict COVID-19 Rapidly Using Chest
}

\section{Images}

\author{
Ranjit Kumar Shing, Sohel Rana, Md. Rakibul Basher, Md. Sazzad Hossain, Md. Hasibul Hasnat, Faisal Ahahmmad \\ Department of Computer Science and Engineering, Bangladesh University of Business and Technology, Dhaka, Bangladesh
}

\begin{abstract}
In December 2019 the novel coronavirus which first appeared in Wuhan City of China spread rapidly around the world and became a pandemic. It has caused a devastating effect on daily lives, public health, and the global economy. As soon as possible we have to detect the affected patient and quickly treat them. There are no accurate automated toolkits available so the need for auxiliary diagnostic tools has increased. Modern outcomes attained using radiology imaging systems recommend that such images have salient evidence about the COVID-19 virus. Real-time reverse transcription-polymerase chain reaction (RT-PCR) is the most common test technique currently used for COVID-19 diagnosis that is too much time-consuming. Using artificial intelligence (AI) techniques associated with radiological imaging can be helpful for the accurate detection of this disease and can also be assistive to overcome the problem of an absence of specialized doctors in remote communities. In this paper, a new model based on Convolutional Neural Network (CNN) that automatically detects COVID-19 using chest images is presented. The proposed model is designed to provide accurate diagnostics for binary classification. A computer vision is rapidly relieved day by day. During our study, we observed that most of the affected people have no common symptoms before checkup COVID-19. If the detection results are incorrect, the patient will not be able to understand that he or she has Covid-19. The proposed model is evaluated by Python libraries namely TensorFlow and Keras. In the proposed model, we got $95 \%$ accuracy as well as the detection of COVID-19 is fast.
\end{abstract}

Keywords: CNN, Covid-19 affected dataset, Chest Images, Python, TensorFlow.

\section{INTRODUCTION}

A present Covid-19 is the most dangerous name. Covid-19 $\mathrm{A}_{\text {is a large family of viruses that start to cause illness }}$ beginning from the common cold to more severe diseases such as the Middle East Respiratory Syndrome (MERS-CoV) and Severe Acute Respiratory Syndrome (SARS-CoV). The major problem of Covid-19 is identification because it is a new strain that has not been previously identified in the human body or animals. A new model for Convolution Neural Network (CNN) that automatically detects COVID-19 using raw chest images is presented. The proposed model is designed to provide accurate diagnostics for binary classification. Computer vision is expressing rapidly day-by-day. Our main target to identify Covid-19 easily.

\section{A. Problem Statement}

We know at present Covid-19 is the main issue the entire world. Many researchers work hard to find out the strain of Covid-19. We use CNN in chest $\mathrm{x}$-ray images to find easily a covid-19 person. Many researchers work on new technique algorithms. CNN is among them. It is challenging for us to get better results in this dataset. We know Covid19 change his action day by day so this is difficult for us to identify the common pattern of this virus. However, if we can success to find out the Covid19 action then we overcome this disease. There are some common challenges to identify Covid-19 like lack of awareness, difficult to find out strain, geographic separation etc. In this paper, A CNN based model is proposed to classify the early stage of Covid-19 using chest X-ray images.

\section{B. Motivation}

Now the whole world is facing the most dangerous situation. Many people die early Covid-19 because we cannot identify this in the early stage. So we think we research Covid19 and trying to identify it in the early stage. We know many researchers already work in this field. We are motivated by them and want to research this field. We add some features and try to hard solve this problem

\section{Significance of the research}

We focus on chest image because Clinical setups and population surveillance employ serology for the detection of antibodies. The limited availability of the test kits makes it challenging to detect every individual affected by the virus. Our research can help the medical site by deciding and give treatment to patients. A doctor can easily find out a Covid-19 person by using chest X-ray images.

\section{Research Contribution}

The contributions of our research:

- We focus on early detection of Covid-19 which is a good solution to save lives of people.

- We modified an existing method to achieve the best accuracy to detect COVID-19.

- We used the some mathematical primitives which increase performance of the proposed CNN model. 


\section{E. Paper Organization}

The rest part of this paper organized as follows: Chapter II highlights the background and literature review on the field of the speaker recognition system. Chapter III contains the proposed architecture of the speaker recognition system, along with a detailed walkthrough of the overall procedures. Chapter IV includes the details of the tests and evaluations that were performed to evaluate our proposed architecture. Chapter V illustrates the analysis of the result. Finally, chapter VI contains the overall conclusion of proposed model.

\section{LITERATURE RIVIEW}

We studied more than thirty related popular CNN based classification algorithms like K-nearest, Native bays, CNN, KNN,BP, XGBoost, BPNN, LR, etc. Also, there have many datasets for the research purpose. By using deep learning manner, researchers acquire more exactness and most of them focus on CNN. They got a better outcome by using CNN manner. Most researchers used chest images for their experiments.

Zhao et al. [1] not only found ground-glass opacities (GGO) or mixed GGO in most of the patients, but they also observed a consolidation, and vascular dilation in the lesion.

$\mathrm{Li}$ and Xia [2] reported GGO and consolidation, interlobular septal thickening and air bronchogram sign, with or without vascular expansion, as common CT features of COVID-19 patients. Peripheral focal or multifocal GGO affecting both lungs in 50\%-75\% of patients is another observation.

$\mathrm{Zu}$ et al. [3] and Chung et al. [4] discovered that 33\% of chest CTs can have rounded lung opacities..

Narin et al. [6] achieved a 98\% COVID-19 detection accuracy using chest X-ray imagescoupled with the ResNet50 model.

Sun et al.[7] concluded that the CNN represented higher performance than deep belief network (DBN) and stacked denoising autoencoder (SDAE) in diagnosing malignant lung nodules with an area under the curve (AUC) of 0.899

Barbosa et al.[8] compared the quantitative CT (qCT), pulmonary function testing (PFT), and semi-quantitative image scores (SQS) metrics and found that pul - monary function testing and qCT metrics demonstrated the highest ac - curacy for monitoring bi- and unilateral lung transplantation with an AUC of 0.771 and 0.817

M. Alhanahnah [9] They focused on two model, Back Propagation Neural Network (BPNN) model and the Logistic Regression (LR) model. LR model give a result with the value between zero to one which indicate the risk factor of this disease. They get higher accuracy in LR algorithm.
Bolei at el[10] Worked with histopathological image. Worked with deep learning algorithms, they used DeNet and SaNet approach where DeNet is used to find out the most useful patches from images and it is use for classification. Using BreakHis dataset and they get 98 percent accuracy.

Wang at el[11] worked with mammography images. Used CNN in detection phase and US_ELM for feature extraction and clustering. In diagnosis phase they use deep feature set of each mammogram as a input of ELM for classification. The output indicates benign or a breast tumor.

TingSim[12] worked with self-regulated multilayer perceptron neural network (ML-NN). This algorithm can help medical experts in diagnosis of breast cancer. This algorithm can classified the input medical images as benign, malignant or normal patient with accuracy, specificity, sensitivity and AUC of $90.59 \%, 90.67 \%, 90.53 \%$, and $0.906 \pm 0.0227$ respectively.

XiaofeErik at el[13] worked with the 2D mammograms and 3D tomosynthesis images. Used CNN for classification. They get better result in 3D tomosynthesis images.

PinarSelma at el[14] worked with mammogram images. Used CNN for classification. Worked with MIAS and BCDR database. Get $87 \%$ accuracy with MIAS database and $88 \%$ accuracy with BCDR database.

Burak Akbugday [15] analyzes different machine classifiers like k-Nearest Neighbors (k-NN), Naïve Bayes(NB) and Support Vector Machine (SVM) with Weka software. The dataset contains 699

Xiaofei Zhamg'[16] el at developing and evaluated a number of $\mathrm{CNN}$ models for whole image classification mammogram. He showed that CNN model he had built and optimized via data augmentation and transfer learning have a great potential for automatic breast cancer detection.

Erwin Halim [17] applied on the different models for early detection of breast cancer like DWT- based multi-resolution MRF (MMRF) segmentation for mammography, MLP for histologic examination, and k-SVMRFE method for gene identification.he expected to increase the accuracy in early detection of breast cancer.

Hanij et al [18] work with thermal image. Use LSVM and $\mathrm{CNN}$ for processing the images. Implement random walks algorithm. Dataset contain 200 images. Get $90.5 \%$ accuracy.

khanAroma[19] work with thermal image. Use SVM classifier. Work with 50 image. Get $84.5 \%$ accuracy.

NanKrzysztof at el[20] In this paper they worked with Breast density classification which is an essential part of breast cancer screening. They apply CNN. Get $88 \%$ accuracy.

TasleemWang at el[21] Worked with histology images. Apply CNN for classification. Worked with ICIAR 2018 breast histology dataset. Get $94.3 \%$ accuracy on 4-class and 97.5\% 2-class histology image. 
Some research papers reviewed above, especially CNN, SVM, ML, XGBOOST algorithms designed to detect Covid19 disease. The researcher obtained better accomplishment with the use of Convolution Neural Network which is flourished on deep learning. In this paper we modified existing CNN based approach to achieve highest accuracy to detect COVID -19 using chest images.

\section{PROPOSED MODEL}

This chapter illustrates the action-ability of the Covid-19 system and the acceptance of demand for the proposed model. Ultimately this chapter clarifies the models of all-inclusive architecture which is elaborated in details.

\section{A. Requirement Analysis}

We implemented the proposed architecture with the following tools and software

- High-performance computing Device.

- Google Colab.

- Open source software libraries for scientific computations like TensorFlow of Python.

- Open source software libraries for machine learning models.

\section{B. Internal Architecture}

This section illustrates the architecture of the proposed Covid-19 classification using the deep learning method. The overall architecture uses the CNN of the features as well. Also, the methodology of the proposed model is exhibited by backto-back explanations comprehensively.

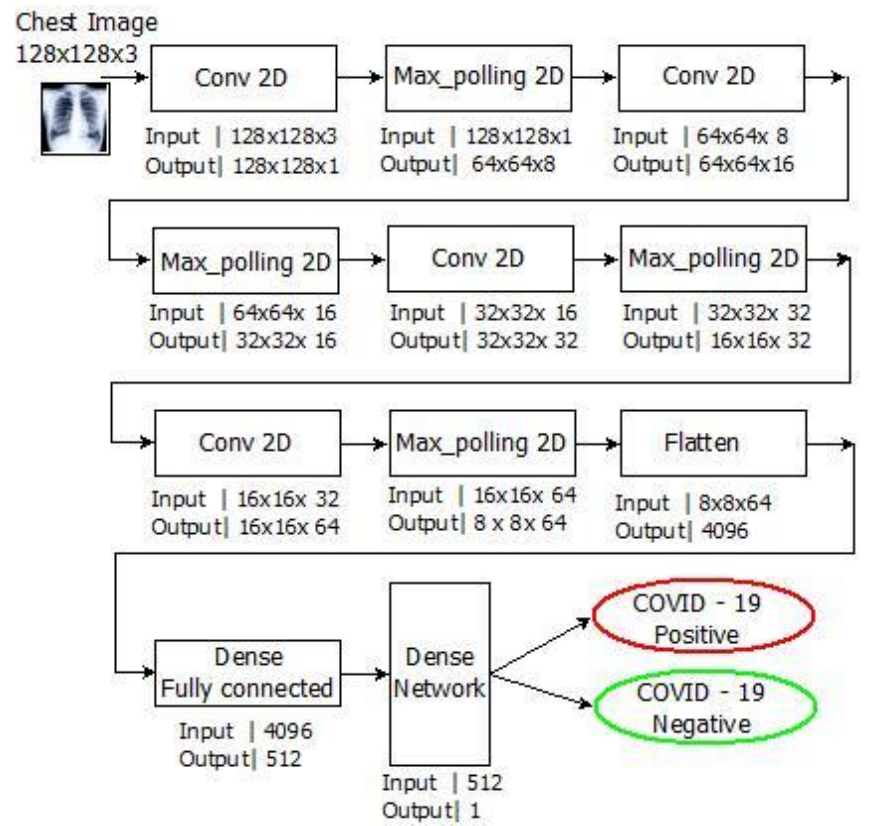

Fig. 1. Internal architecture

In the proposed approach, we used the $\mathrm{CNN}$ features to achieve better accuracy.The proposed model consists of four CNN layers namely Convo 2D, Max_pooling2D, Flatten, and
Dense layer). We used four Convo2D and two Dense layers. These covo2D are $(8,16,32,64)$ and Dense are $(512,1)$. The size of Maxpooling2D used in the model is $2 \times 2$ with same padding . A flatten layer is used to create a single long feature vector size of 4096. The aactivation functions used in the dense layers are ReLu and Sigmoid. The activation function ReLu is applied to the final dense layer $(512 \times 1)$ to classify the Covid 19 in binary manner (positive or negative). For fully connected $4096 \times 512$ neural network, we used sigmoid function as the activation function.

The fully connected layer is passed to the ReLU layer so as to normalize the binary classification vector. After the output of the classification vector, two categories i.e. Covid-19 positive or Negative for evaluation. All the hidden layers use sigmoid as their activation function. The sigmoid function is more computationally efficient because it leads to faster learning and it also decreases the likelihood of vanishing gradient problem.

\section{DFD of the model}

This is the data flow diagram of the proposed model.

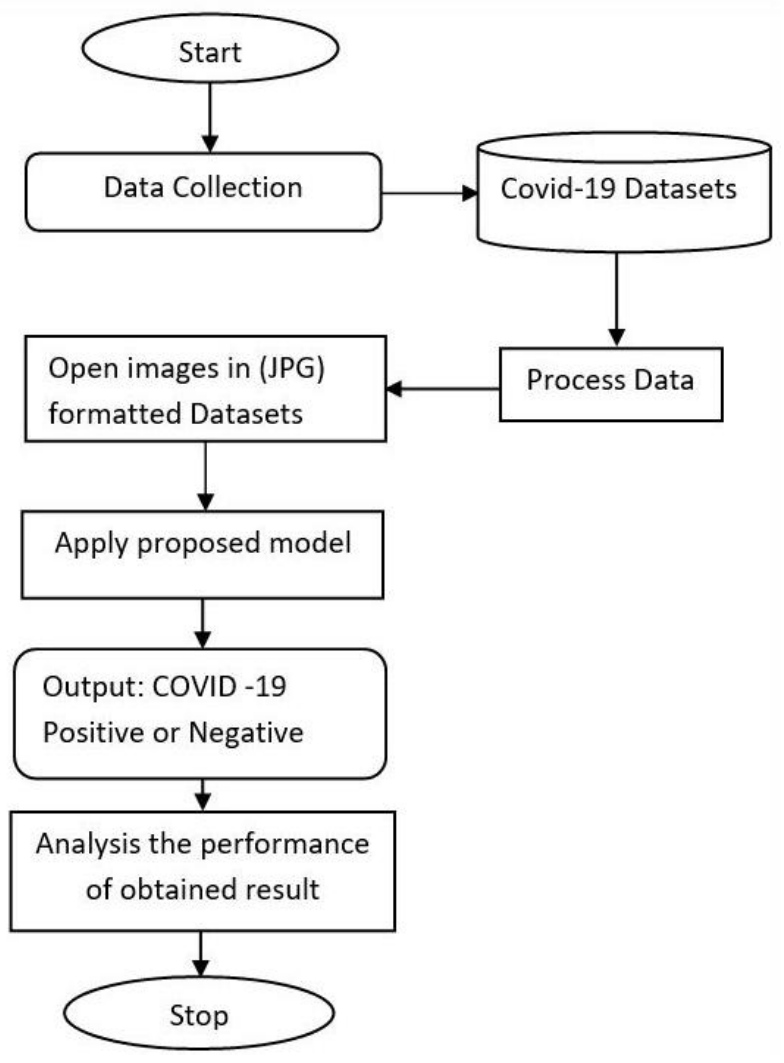

Fig. 2. DFD of the model

This section illustrates the Data Flow Diagram of the proposed models' working process. After collecting dataset, proposed model is trained to predict the Covid-19 virus from images. We used MINI-MIAS dataset to train the model. There is a performance evaluation process before making the final decision. 


\section{EXPERIMENTAL SETUP}

The proposed model is implemented by using Python language and Google collaborator platform. The Convolutional neural network models are implemented using python. Also, we used NumPy and Pandas for additional calculation and implementation purpose. The visual evolution reports were produced using Matplotlib in python. At the same time, this section illustrates the evaluation metrics used to measure the accuracy of the results and the detailed analysis of the results.

\section{A. Configuration of dataset for the model}

In the proposed model, we used the batch size of the dataset as 64 . Size of chest image is $128 \times 128$ as height and width. The image batch of our dataset is ("64", " 128 ", " 128 ", and " 3 "). There are two important methods namely cache, prefetch which are used to load the datasets for the proposed model.

\section{B. Data Pre-processing}

Data pre-processing in any machine learning process is the step where data is converted or encoded, bringing it to the point where the machine can now easily parse. In other words, the data properties can be easily explained by algorithms. For training and evaluation, we used the MINI-MIAS dataset. We use two classes for trains and validation. After pre-processing chest images is shown below.
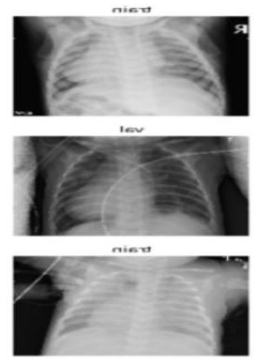
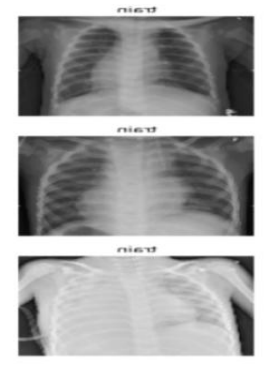

Fig. 3. Data visualization after pre-processing

\section{Data Augmentation}

Data augmentation creates new training data from existing training data. Cropping, padding, and horizontal flipping are commonly used to train large neural networks for data augmentation techniques. Using the augmentation, the model gets the better performance to finding accuracy.
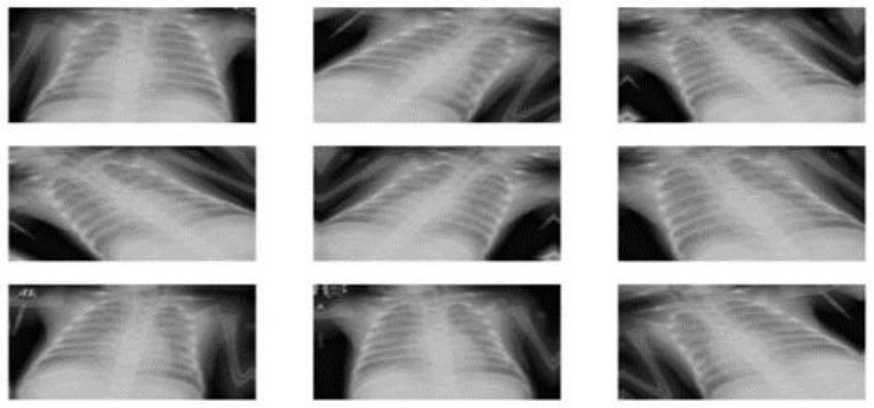

Fig. 4. Data visualization after augmentation

\section{Result of the model}

We designed the different layers of the proposed model as shown below.

\begin{tabular}{|c|c|c|}
\hline Layer (type) & Output Shape & Param \# \\
\hline $\begin{array}{l}=========================== \\
\text { conv } 2 \mathrm{~d}(\text { Conv2D) }\end{array}$ & $\begin{array}{l}===================+ \\
\text { (None, } 128,128,8)\end{array}$ & $\begin{array}{l}=====+1 \\
224\end{array}$ \\
\hline max_pooling2d (MaxPooling2D) & (None, $64,64,8$ ) & 0 \\
\hline conv2d_1 (Conv2D) & (None, $64,64,16$ ) & 1168 \\
\hline max_pooling2d_1 (MaxPooling2 & (None, 32, 32, 16) & 0 \\
\hline conv2d_2 (Conv2D) & (None, 32, 32, 32) & 4640 \\
\hline max_pooling2d_2 (MaxPooling2 & (None, $16,16,32$ ) & 0 \\
\hline conv2d_3 (Conv2D) & (None, $16,16,64$ ) & 18496 \\
\hline max_pooling2d_3 (MaxPooling2 & (None, $8,8,64$ ) & 0 \\
\hline flatten (Flatten) & (None, 4096) & 0 \\
\hline dense (Dense) & (None, 512) & 2097664 \\
\hline dense_1 (Dense) & (None, 1) & 513 \\
\hline
\end{tabular}

Total params: 2,122,705

Trainable params: 2,122,705

Non-trainable params: 0

Fig. 5. Proposed CNN Mode

\section{E. Epoch's Result}

We got the following result for the proposed model.

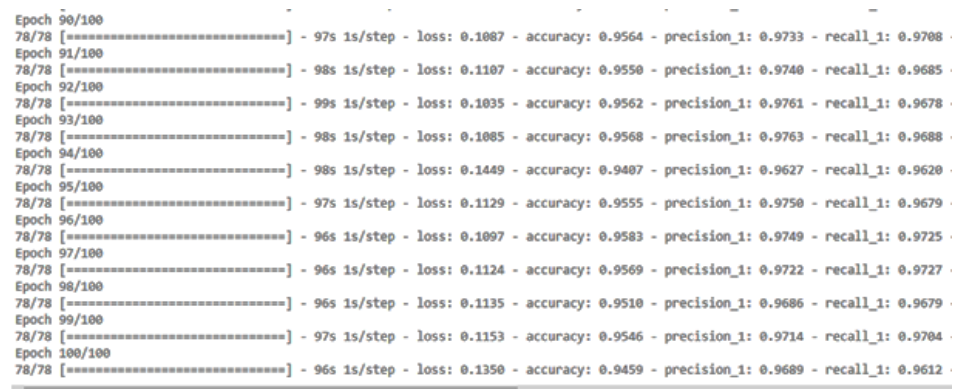

Fig. 6. Epoch's Result_1

true_positives_1: 1957.5696 - true_negatives_1: 495.2911 - false_positives_1: 43.3797 - false_negatives_1: 52.8354 true_positives_1: 1926.3924 - true_negatives_1: 500.8228 - false_positives_1: 49.6835 - false_negatives_1: 57.8987 true_positives_1: 1926.2785 - true_negatives_1: 491.6962 - false_positives_1: 56.1013 - false_negatives_1: 64.8861 true_positives_1: 1917.1646 - true_negatives_1: 506.0000 - false_positives_1: 47.2785 - false_negatives_1: 64.3544 true_positives_1: 1925.0506 - true_negatives_1: 494.1266 - false_positives_1: 51.5570 - false_negatives_1: 62.8734 true_positives_1: 1929.9241 - true_negatives_1: 481.8861 - false_positives_1: 64.1772 - false_negatives_1: 72.4937 true_positives_1: 1905.0380 - true_negatives_1: 502.4557 - false_positives_1: 49.9494 - false_negatives_1: 61.8861 true_positives_1: 1951.1899 - true_negatives_1: 483.7595 - false_positives_1: 50.9241 - false_negatives_1: 55.4684 true_positives_1: 1923.0633 - true_negatives_1: 496.5696 - false_positives_1: 54.9367 - false_negatives_1: 57.8481 true_positives_1: 1923.9367 - true_negatives_1: 501.4304 - false_positives_1: 55.7848 - false_negatives_1: 63.1646 true_positives_1: 1918.6582 - true_negatives_1: 494.5443 - false_positives_1: 51.9367 - false_negatives_1: 60.7342 true_positives_1: 1910.7975 - true_negatives_1: 502.0253 - false_positives_1: 60.0759 - false_negatives_1: 74.9873

Fig. 7. Epoch's result_2 


\section{Performance ANALysis AND Discussion}

Accuracy, Recall and Precision are the core metrics for evaluating a classification model in CNN. Informally, accuracy is a fraction of what our model accurately estimates. In general, accuracy has the following definitions.

$$
\text { Accuracy }=\frac{\text { Numberofcorrectpredictions }}{\text { Totalnumberofpredictions }}
$$

For binary classification, accuracy can also be calculated in terms of positives and negatives as follows:

$$
\begin{aligned}
& \text { Accuracy }=\frac{T P+T N}{T P+T N+F P+F N} \\
& \text { Recall }=\frac{T P}{F N+T P}
\end{aligned}
$$

And

$$
\text { Precision }=\frac{T P}{F P+T P}
$$

where $T P=$ True Positives, $T N=$ True Negatives, $F P=$ False Positives, and $F N=$ False Negatives.

A. Now Accuracy for proposed model $=\frac{T P+T N}{T P+T N+F P+F N}$

$$
\begin{aligned}
& =\frac{1910.7975+502.0253}{1910.7975+502.0253+60.0759+74.9873} \\
& =0.95 * 100 \\
& =95 \%
\end{aligned}
$$

B. Matrix of the Recall for proposed model $=\frac{T P}{F N+T P}$

$$
\begin{aligned}
& =\frac{1910.7975}{74.9873+1910.7975} \\
& =0.96 * 100 \\
& =96 \%
\end{aligned}
$$

C. Matrix of the precession $=\frac{T P}{F P+T P}$

$$
\begin{aligned}
& =\frac{1910.7975}{60.0759+1910.7975} \\
& =0.97 * 100 \\
& =97 \%
\end{aligned}
$$

D. Matrix of the F1 Score $=\frac{2 *(0.97 * 0.96)}{0.97+0.96}$

$$
\begin{aligned}
& =0.96 * 100 \\
& =96 \%
\end{aligned}
$$

TABLE I. PERFORMANCE METRICS

\begin{tabular}{|c|c|c|c|}
\hline Accuracy $(\%)$ & Recall (\%) & Precision $(\%)$ & FI Score $(\%)$ \\
\hline 95 & 96 & 97 & 96 \\
\hline
\end{tabular}

From the evaluation reports, it is evident that this CNN architecture performs the most satisfying accuracy on Covid19 detection tasks.

\section{CONCLUSION AND FUTURE WORKS}

Nowadays COVID-19 classification with chest images using $\mathrm{CNN}$ is going to rapidly popular. This work is helpful because this work's performance is much better than the others. Our work has $95 \%$ accuracy. Hope that this work will be efficient in diagnosis in medical. It will be faster to identify COVID-19 virus and helps peoples to save lives.

In future, we will test the model for more datasets to increase the performance using the most powerful hardware.

\section{REFERENCS}

[1] X. Wei, Y. Chen and Z. Zhang, "Comparative Experiment of Convolutional Neural Network (CNN) Models Based on Pneumonia X-ray Images Detection," 2020 2nd International Conference on Machine Learning, Big Data and Business Intelligence (MLBDBI), Taiyuan, China, 2020, pp. 449-454, doi: 10.1109/MLBDBI51377.2020.00095.

[2] B. K. Umri, M. Wafa Akhyari and K. Kusrini, "Detection of Covid-19 in Chest X-ray Image using CLAHE and Convolutional Neural Network," 2020 2nd International Conference on Cybernetics and Intelligent System (ICORIS), Manado, Indonesia, 2020, pp. 1-5, doi: 10.1109/ICORIS50180.2020.9320806.

[3] S. Deep Deb and R. Kumar Jha, "COVID-19 detection from chest X-Ray images using ensemble of CNN models," 2020 International Conference on Power, Instrumentation, Control and Computing (PICC), Thrissur, India, 2020, pp. 1-5, doi: 10.1109/PICC51425.2020.9362499.

[4] B. Jabber, J. Lingampalli, C. Z. Basha and A. Krishna, "Detection of Covid-19 Patients using Chest X-ray images with Convolution Neural Network and Mobile Net," 2020 3rd International Conference on Intelligent Sustainable Systems (ICISS), Thoothukudi, India, 2020, pp. 1032-1035, doi: 10.1109/ICISS49785.2020.9316100.

[5] A. A. w. A. Musleh and A. Y. Maghari, "COVID-19 Detection in X-ray Images using CNN Algorithm," 2020 International Conference on Promising Electronic Technologies (ICPET), Jerusalem, Palestine, 2020, pp. 5-9, doi: 10.1109/ICPET51420.2020.00010.

[6] A. M. Fangoh and S. Selim, "Using CNN-XGBoost Deep Networks for COVID-19 Detection in Chest X-ray Images," 2020 15th International Conference on Computer Engineering and Systems (ICCES), Cairo, Egypt, 2020, pp. 1-7, doi: 10.1109/ICCES51560.2020.9334600.

[7] N. Rashid, M. A. Faisal Hossain, M. Ali, M. I. Sukanya, T. Mahmud and S. A. Fattah, "Transfer Learning Based Method for COVID-19 Detection From Chest X-ray Images," 2020 IEEE REGION 10 CONFERENCE (TENCON), Osaka, Japan, 2020, pp. 585-590, doi: 10.1109/TENCON50793.2020.9293850.

[8] Y. Khan, P. Khan, S. Kumar, J. Singh and R. M. Hegde, "Detection and Spread Prediction of COVID-19 from Chest X-ray Images using Convolutional Neural Network-Gaussian Mixture Model," 2020 IEEE 17th India Council International Conference (INDICON), New Delhi, India, 2020, pp. 1-6, doi: 10.1109/INDICON49873.2020.9342159.

[9] E. Irmak, "A Novel Deep Convolutional Neural Network Model for COVID-19 Disease Detection," 2020 Medical Technologies Congress (TIPTEKNO), Antalya, Turkey, 2020, pp. 1-4, doi: 10.1109/TIPTEKNO50054.2020.9299286.

[10] S. Tewari, U. Agrawal, S. Verma, S. Kumar and S. Jeevaraj, "Ensemble Model for COVID-19 detection from chest X-ray Scans using Image Segmentation, Fuzzy Color and Stacking Approaches," 2020 IEEE 4th Conference on Information \& Communication Technology (CICT), Chennai, India, 2020, pp. 16, doi: 10.1109/CICT51604.2020.9312076.

[11] T. Karlita, E. M. Yuniarno, I. K. E. Purnama and M. H. Purnomo, "Detection of COVID-19 on Chest X-Ray Images using Inverted Residuals Structure-Based Convolutional Neural Networks," 2020 
3rd International Conference on Information and Communications Technology (ICOIACT), Yogyakarta, Indonesia, 2020, pp. 371376, doi: 10.1109/ICOIACT50329.2020.9332153.

[12] S. Sakib, T. Tazrin, M. M. Fouda, Z. M. Fadlullah and M. Guizani, "DL-CRC: Deep Learning-Based Chest Radiograph Classification for COVID-19 Detection: A Novel Approach," in IEEE Access, vol. 8, pp. 171575-171589, 2020, doi: 10.1109/ACCESS.2020.3025010.

[13] Y. Yari, T. V. Nguyen and H. Nguyen, "Accuracy Improvement in Detection of COVID-19 in Chest Radiography," 2020 14th International Conference on Signal Processing and Communication Systems (ICSPCS), Adelaide, SA, Australia, 2020, pp. 1-6, doi: 10.1109/ICSPCS50536.2020.9310066.

[14] R. Bhadra and S. Kar, "Covid Detection from CXR Scans using Deep Multi-layered CNN," 2020 IEEE Bombay Section Signature Conference (IBSSC), Mumbai, India, 2020, pp. 214-218, doi: 10.1109/IBSSC51096.2020.9332210.

[15] D. Haritha, M. K. Pranathi and M. Reethika, "COVID Detection from Chest X-rays with DeepLearning: CheXNet," 2020 5th International Conference on Computing, Communication and Security (ICCCS), Patna, India, 2020, pp. 1-5, doi: 10.1109/ICCCS49678.2020.9277077.

[16] A. Waheed, M. Goyal, D. Gupta, A. Khanna, F. Al-Turjman and P. R. Pinheiro, "CovidGAN: Data Augmentation Using Auxiliary Classifier GAN for Improved Covid-19 Detection," in IEEE Access, vol. 8, pp. 91916-91923, 2020, doi: 10.1109/ACCESS.2020.2994762.

[17] M. K. Nath, A. Kanhe and M. Mishra, "A Novel Deep Learning Approach for Classification of COVID-19 Images," 2020 IEEE 5th International Conference on Computing Communication and Automation (ICCCA), Greater Noida, India, 2020, pp. 752-757, doi: 10.1109/ICCCA49541.2020.9250907.

[18] I. Mporas and P. Naronglerdrit, "COVID-19 Identification from Chest X-Rays," 2020 International Conference on Biomedical Innovations and Applications (BIA), Varna, Bulgaria, 2020, pp. 69-72, doi: 10.1109/BIA50171.2020.9244509.

[19] E. -S. M. El-Kenawy et al., "Advanced Meta-Heuristics, Convolutional Neural Networks, and Feature Selectors for Efficient COVID-19 X-Ray Chest Image Classification," in IEEE Access, vol. 9, pp. 36019-36037, 2021, doi: 10.1109/ACCESS.2021.3061058.

[20] M. M. R. Khan et al., "Automatic Detection of COVID-19 Disease in Chest X-Ray Images using Deep Neural Networks," 2020 IEEE 8th R10 Humanitarian Technology Conference (R10-HTC), Kuching, Malaysia, 2020, pp. 1-6, doi: 10.1109/R10HTC49770.2020.9357034.

[21] O. Saha, J. Tasnim, M. T. Raihan, T. Mahmud, I. Ahmmed and S. A. Fattah, "A Multi-Model Based Ensembling Approach to Detect COVID-19 from Chest X-Ray Images," 2020 IEEE REGION 10 CONFERENCE (TENCON), Osaka, Japan, 2020, pp. 591-595, doi: 10.1109/TENCON50793.2020.9293802.

[22] S. V. Militante, N. V. Dionisio and B. G. Sibbaluca, "Pneumonia and COVID-19 Detection using Convolutional Neural Networks," 2020 Third International Conference on Vocational Education and Electrical Engineering (ICVEE), Surabaya, Indonesia, 2020, pp. 16, doi: 10.1109/ICVEE50212.2020.9243290.

[23] U. Singh, A. Totla and D. P. Kumar, "Deep Learning Model to Predict Pneumonia Disease based on Observed Patterns in Lung X-rays," 2020 4th International Conference on Electronics, Communication and Aerospace Technology (ICECA), Coimbatore, India, 2020, pp. 1315-1320, doi: 10.1109/ICECA49313.2020.9297388.

[24] I. Ahmed, A. Ahmad and G. Jeon, "An IoT based deep learning framework for early assessment of Covid-19," in IEEE Internet of Things Journal, doi: 10.1109/JIOT.2020.3034074.

[25] Sohel Rana, Wadud, Ali Azgar, Dr. M Abul Kashem, "A Survey Paper of Lightweight Block Ciphers Based on Their Different Design Architectures and Performance Metrics" International Journal of Computer Engineering and Information Technology, June 2019, Volume 11, Issue 6.
[26] Sohel Rana, M. Rubaiyat Hossain Mondal and A. H. M. Shahariar Parvez, "A New Key Generation Technique based on Neural Networks for Lightweight Block Ciphers" International Journal of Advanced Computer Science and Applications(IJACSA), 12(6), 2021. 